\title{
Multispectral sensing of biological liquids with hollow-core microstructured optical fibres
}

\author{
Timur Ermatov ${ }^{1}$, Roman E. Noskov $\mathbb{1}^{2,3}$, Andrey A. Machnev ${ }^{2,3}$, Ivan Gnusov ${ }^{1}$, Vsevolod Atkin ${ }^{4}$, Ekaterina N. Lazareva ${ }^{4,5}$, \\ Sergei V. German', Sergey S. Kosolobov' ${ }^{1}$, Timofei S. Zatsepin ${ }^{1,6}$, Olga V. Sergeeva', Julia S. Skibina ${ }^{7}$ Pavel Ginzburg ${ }^{2,3,8}$, \\ Valery V. Tuchin ${ }^{4,5,9}$, Pavlos G. Lagoudakis (1D) and Dmitry A. Gorin (1) ${ }^{1}$
}

\begin{abstract}
The state of the art in optical biosensing is focused on reaching high sensitivity at a single wavelength by using any type of optical resonance. This common strategy, however, disregards the promising possibility of simultaneous measurements of a bioanalyte's refractive index over a broadband spectral domain. Here, we address this issue by introducing the approach of in-fibre multispectral optical sensing (IMOS). The operating principle relies on detecting changes in the transmission of a hollow-core microstructured optical fibre when a bioanalyte is streamed through it via liquid cells. IMOS offers a unique opportunity to measure the refractive index at 42 wavelengths, with a sensitivity up to $\sim 3000 \mathrm{~nm}$ per refractive index unit (RIU) and a figure of merit reaching $99 \mathrm{RIU}^{-1}$ in the visible and near-infra-red spectral ranges. We apply this technique to determine the concentration and refractive index dispersion for bovine serum albumin and show that the accuracy meets clinical needs.
\end{abstract}

\section{Introduction}

The increasing medical need for robust techniques suitable for real-time diagnostics at the place of patient care is at the cutting edge of modern biosensing ${ }^{1}$. Among the variety of available sensing devices, optical label-free sensors demonstrate high sensitivity to ambient refractive index (RI) variations, attracting considerable attention from the chemical, biomedical and food processing industries ${ }^{2}$. The main efforts in the development of such sensors are focused on increasing RI sensitivity (RIS) through the employment of cavity resonances (including surface plasmon resonances, Mie resonances, whispering gallery modes, etc.) and propagating eigenmodes in dielectric and plasmonic nanostructures ${ }^{3}$. Conceptually, another approach is realized by hollow-core microstructured optical fibres (HC-MOFs), which allow sensing

\footnotetext{
Correspondence: Dmitry A. Gorin (d.gorin@skoltech.ru)

'Skolkovo Institute of Science and Technology, 3 Nobelya str., Moscow 121205, Russia

${ }^{2}$ Department of Electrical Engineering, Tel Aviv University, Ramat Aviv, Tel Aviv 69978, Israel

Full list of author information is available at the end of the article

These authors contributed equally: Timur Ermatov, Roman E. Noskov
}

of liquid analytes by monitoring the changes in the transmission; HC-MOFs have a great advantage of enabling high volume for measuring light-analyte interactions, which improves the RIS in comparison to that with cavity-based counterparts ${ }^{4}$.

Almost all optical sensors typically exploit a single resonance feature in the reflection/transmission/scattering spectra, following resonant shifts, associated with variations in the analyte RI and concentration ${ }^{5}$. Along with the specificity to target biomolecules supplied by functionalization of sensing template nanostructures with antibodies, aptamers and other analyte binders ${ }^{6}$, such biosensors may show very high sensitivity and figures of merit $^{7,8}$. However, this strategy is time-consuming and quite expensive, and it disregards RI optical dispersion, which can act as a simple and cost-effective fingerprint of liquid biosamples to enable real-time monitoring of changes in their composition. In particular, variations in the optical dispersion of blood serum may indicate some diseases since it is directly related to changes in blood components, specifically the concentration of albumin and the appearance of its conjugated forms ${ }^{9-12}$. Recently,

\section{(c) The Author(s) 2020}

(c) (i) Open Access This article is licensed under a Creative Commons Attribution 4.0 International License, which permits use, sharing, adaptation, distribution and reproduction c. in any medium or format, as long as you give appropriate credit to the original author(s) and the source, provide a link to the Creative Commons license, and indicate if changes were made. The images or other third party material in this article are included in the article's Creative Commons license, unless indicated otherwise in a credit line to the material. If material is not included in the article's Creative Commons license and your intended use is not permitted by statutory regulation or exceeds the permitted use, you will need to obtain permission directly from the copyright holder. To view a copy of this license, visit http://creativecommons.org/licenses/by/4.0/. 


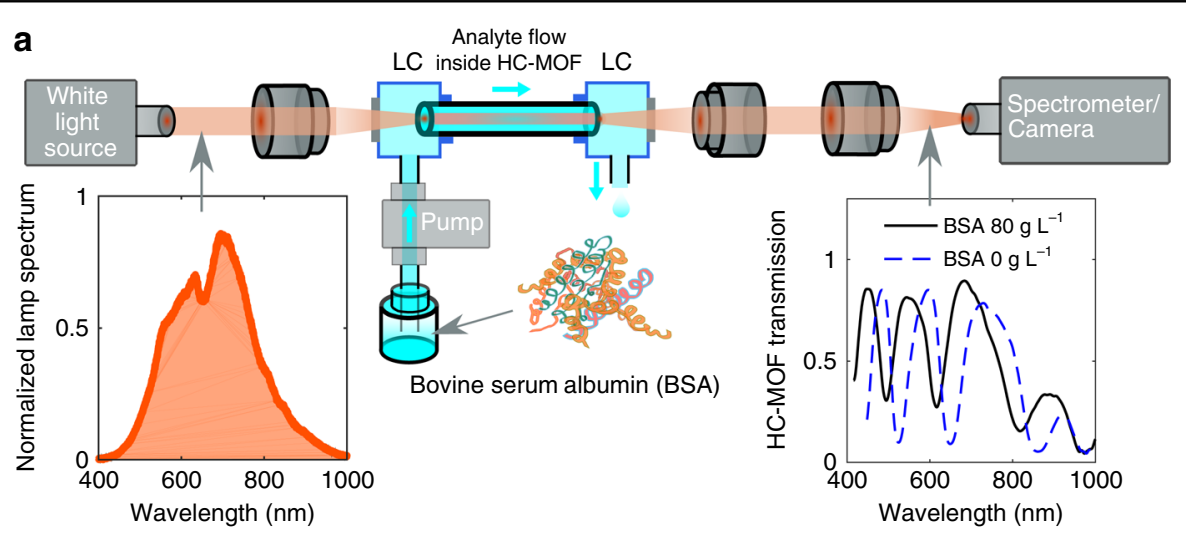

b

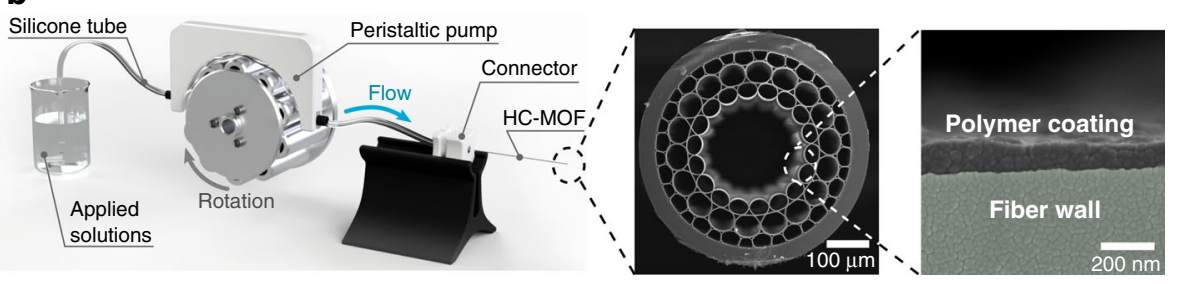

Fig. 1 Illustration of the dynamic multispectral sensing concept for liquid samples. a Scheme of the setup for transmission characterization of HC-MOFs. Fibre facets are fastened in liquid cells (LCs), which are optically accessible via thin glass windows, allowing simultaneous pumping of fluids through fibre capillaries and measurement of the transmission spectrum. The red rays illustrate the light path from a broadband halogen lamp through the HC-MOF to the spectrometer and the CCD (charge-coupled device) camera to record the output mode profile. The insets depict the input and output spectra for different concentrations of BSA dissolved in PBS. b The setup for functionalization of HC-MOFs with LbL assembly. A peristaltic pump drives the flow of applied solutions (polyelectrolyte water solution and pure water) through the full fibre length, leading to the formation of the polymer coating on the inner surface of the core capillary. The procedure is repeated with inversely charged polyelectrolytes to create the desired number of bilayers. The insets depict the scanning electron microscopy (SEM) images of the fibre end face and the magnified capillary wall with the polymer coating formed by 18 PE bilayers

it was shown that variations in the blood serum RI could be used as an additional criterion in the analysis of antitumour therapy ${ }^{13,14}$, and RI monitoring for glycated haemoglobin and albumin enables diagnostics of type 2 diabetes and pre-diabetic status ${ }^{15,16}$.

Serum albumin is the most abundant blood plasma protein and plays a pivotal role in maintaining oncotic pressure as well as in transporting poorly soluble molecules, including lipid-soluble hormones, bile salts, unconjugated bilirubin and many others ${ }^{17}$. The normal concentration of albumin in the serum of human adults is $35-54 \mathrm{gL}^{-1}$, while its deviations indicate various abnormal conditions and diseases ${ }^{12}$. Since albumin is optically transparent, direct determination of the analyte concentration by measuring the optical absorption is not applicable. The typical detection of the albumin concentration is based on the changes in dye absorbance (such as bromocresol green or bromocresol purple) upon binding to albumin ${ }^{18}$. Alternatively, it was proposed to detect low concentrations of albumin with the optical spring effect in an optomechanical oscillator ${ }^{19}$ and plasmon polaritons in a hyperbolic metamaterial ${ }^{7}$. Such techniques, however, are time-consuming and do not allow instantaneous monitoring of albumin in biological fluids in real time.

Here, we introduce the concept of in-fibre multispectral optical sensing (IMOS) for liquid biological samples in both static and real-time modes. The sensing principle relies on detecting spectral shifts of maxima and minima in the transmission spectrum of a hollow-core microstructured optical fibre when a liquid bioanalyte is streamed through it via specially designed liquid chambers (Fig. 1a). These resonant features are associated with Fabry-Perot resonances in the core capillary wall, and their spectral positions are unambiguously related to the bioanalyte RIs. A single fibre enables measurement of the RI at $\sim 10$ wavelengths with a sensitivity up to $\sim 3000 \mathrm{nmRIU}^{-1}$ and a figure of merit (FOM) reaching 99 $\mathrm{RIU}^{-1}$ in the visible and near-infra-red spectral domains. To increase the number of acquisition wavelengths to 42 , we produce several $\mathrm{HC}-\mathrm{MOF}$ with slightly shifted transmission windows by coating their capillaries with polymer nanofilms of various thicknesses using the highly controllable and reproducible layer-by-layer (LbL) assembly of oppositely charged polyelectrolytes (PEs) (Fig. $1 \mathrm{~b})$. We demonstrate the practical performance of IMOS 
by measuring the concentration of bovine serum albumin (BSA) dissolved in water and in a phosphate-buffered saline (PBS) solution in both static and dynamic modes and show a resolution $\sim 1 \mathrm{gL}^{-1}$ when determining the BSA concentration, which matches the accuracy of standard tests on albumin ${ }^{18}$. Furthermore, for the first time, to our knowledge, we have extracted RI dispersion of pure BSA in a wide wavelength range of $400-850 \mathrm{~nm}$. The important advantage of IMOS in comparison with many other optical biosensors is its simplicity and cost efficiency since it does not require any external cavity or interferometer, and the production of functionalized $\mathrm{HC}$ MOFs is simple and inexpensive. In addition, IMOS makes it possible to perform RI measurements within a wide spectral domain in real time, which is still a challenging issue for other alternative methods.

\section{Results}

Principle of in-fibre multispectral optical sensing (IMOS)

To measure the optical transmission of liquid-filled HCMOFs, we employ the setup shown in Fig. 1a. Liquid filling of fibre capillaries is obtained by inserting the tips of HC-MOFs into small liquid cells (LCs) equipped with tubing interfaces and optically transparent windows ${ }^{4}$. The LCs are 3D-printed from acrylonitrile butadiene styrene and exhibit a dead volume of $\sim 50 \mu \mathrm{L}$ (see the Supplementary information). This value is sufficiently small for stable and reproducible measurements in both static and dynamic regimes (at least at the pumping rate of $\sim 1 \mathrm{~mL}$ $\mathrm{min}^{-1}$ or slower). The flow of the analyte through the HC-MOF is driven by a peristaltic pump (Shenchen LabV1) connected to the inlet of the LC. Microscope objectives (Olympus, 10x) are used to couple the light from a halogen lamp (Thorlabs SLS201L) into the fibre and to collect the transmitted signal. The output signal is analyzed with a spectrometer (Ocean Optics QE Pro) combined with a CCD camera (Thorlabs DCU223C) (see further details in "Materials and methods").

The employed soft-glass HC-MOFs have a considerably thick wall of the central capillary of $1.82 \mu \mathrm{m}$ (see Fig. S1) so that the light guiding mechanism can be described via Fabry-Perot resonances ${ }^{20,21}$. In accord with this model, the resonant coupling between the main core (guiding) mode and the capillary (cladding) modes corresponds to the maxima (Eq. (1)) and minima (Eq. (2)) in the fibre transmission, which occur at

$$
\begin{aligned}
& \lambda_{j \text { max }}=\frac{4 d}{2 j+1} \sqrt{n_{2}^{2}-n_{1}^{2}} \\
& \lambda_{j \text { min }}=\frac{2 d}{j} \sqrt{n_{2}^{2}-n_{1}^{2}}
\end{aligned}
$$

where $j$ is an integer describing the capillary mode order $(j=1,2,3, \ldots), n_{1}$ is the RI of an analyte filling the capillaries, $n_{2}$ is the RI of the fibre glass and $d$ indicates the wall thickness for the first capillary layer. This relation provides a convenient link between the RIs of an analyte and the maxima and minima in the fibre transmission. Specifically, for $d=1.82 \mu \mathrm{m}$, the HC-MOF filled with water exhibits four transmission windows in the wavelength range $400-900 \mathrm{~nm}$, and the minima appearing at 411, 494, 617 and $821 \mathrm{~nm}$ for $j=6,5,43$ obey Eq. (1). However, since the transmission windows can be asymmetric due to non-negligible dispersion of the fibre glass and other optical elements (Fig. 1a), the positions of the centroids have been found to be more sensitive to the variations in an analyte RI than the maxima, and we use them for further measurements.

Thus, a single fibre allows simultaneous measurement of an analyte's RI synchronously at seven wavelengths in the visible and near-infra-red spectral domains. However, such a number of wavelengths is still insufficient for an accurate retrieval of RI optical dispersion, especially in cases when data are contradictory or not available. The number of discrete wavelengths available for measuring RI can be increased by using several HC-MOFs with slightly shifted transmission windows. However, reproducibly drawing such a family of fibres with variations in $d \sim 10 \mathrm{~nm}$ is technically quite a challenging task that would inevitably increase the cost of IMOS. In the next section, we resolve this issue by presenting a simple and inexpensive technique that allows a small increase in the thickness of capillary walls in a reproducible and highly controllable way.

\section{Functionalization of hollow-core microstructured optical fibres with LbL assembly}

The basic principle of our approach is the LbL assembly of oppositely charged PEs onto the glass surface. Figure $1 \mathrm{~b}$ shows the setup used for coating the fibre capillaries ${ }^{22}$. The peristaltic pump creates a flow of PE solutions through the fibre with a controllable and persistent rate for a pre-determined volume. This procedure results in the uniform deposition of PE layers inside the fibre capillaries. As PEs, aqueous solutions of polycationic poly (allylamine hydrochloride) (PAH) and polyanionic poly (styrenesulfonate) (PSS) ${ }^{23}$ have been used.

The coating procedure can be described as follows. First, the HC-MOF is rinsed with deionized water for $2 \mathrm{~min}$ at a flow rate of $500 \mu \mathrm{L} \mathrm{min}{ }^{-1}$ to remove dust particles prior to PE deposition. The very first deposited $\mathrm{PE}$ layer consists of polyethylenimine (PEI) serving as an adhesive or anchor agent that provides a high surface charge density with a homogenous distribution due to its high molecular weight and branched structure ${ }^{24,25}$. For each layer, we coat $\mathrm{HC}-\mathrm{MOF}$ sequentially by $\mathrm{PAH}$ and PSS PE solutions (each at a concentration of $2 \mathrm{mg} \mathrm{mL}^{-1}$ ) for $7 \mathrm{~min}$. To ensure successful adsorption and to prevent 
colloid depletion during the multistep LbL deposition process, we use concentrated PE solutions $\left(2 \mathrm{mg} \mathrm{mL}^{-1}\right)$ that allow exceeding the minimum threshold for molecule attachment and reversing the charge polarity for each adsorbed layer ${ }^{26,27}$. Finally, we remove unbound polymer molecules and prevent cross-contamination of the solutions by washing the samples with pure deionized water after each layer is deposited ${ }^{28}$.

\section{lonic strength influence on the polymer coating thickness}

It has been demonstrated that the thickness of LbLassembled layers on a planar substrate is proportional to the square root of the ionic strength ${ }^{29}$ that, in turn, is the square function of the molar concentration of ions. Hence, the ionic strength is typically supported by sodium and chlorine ions, which are the main components of blood plasma as well as saline solution. Neff et al. ${ }^{29}$ demonstrated that an increase in the buffer molar concentration yields thicker LbL-assembled PAH/PSS multilayers. For example, the thickness per single layer is $1.3 \pm 0.1 \mathrm{~nm}$ at $0.05 \mathrm{M} \mathrm{NaCl}$ but is $2.2 \pm 0.1 \mathrm{~nm}$ at $0.5 \mathrm{M}$ $\mathrm{NaCl}^{29}$. In addition, the higher concentration of sodium chloride gives rise to an increased roughness caused by altering the molecular conformation from a linear to globular structure ${ }^{30}$.

To gain insight into the impact of the solution ionic strength on the coating performance, we systematically compare the thickness and morphology of assembled PAH/PSS films with PEs at the same concentration dissolved in deionized water and in buffer containing $0.15 \mathrm{M}$ $\mathrm{NaCl}$. Figure 2a shows the thickness of the coatings prepared by the alternating deposition of PAH and PSS layers from $\mathrm{PE}$ solutions with and without $\mathrm{NaCl}$ as a function of the number of deposited PE bilayers. The coating thickness linearly depends on the number of assembled PAH/ PSS bilayers. However, the average thickness increase is $1.8 \pm 0.3 \mathrm{~nm}$ and $7.0 \pm 1.3 \mathrm{~nm}$ per bilayer for the salt-free $\mathrm{PE}$ solutions and in the presence of $0.15 \mathrm{M} \mathrm{NaCl}$, respectively. These values differ from previously published data for LbL coating of planar substrates, where the average thickness per PE layer was $\sim 1-3 \mathrm{~nm}^{23,31-33}$. We attribute this discrepancy to different hydrodynamic conditions for PE adsorption in our study ${ }^{22}$ and the effect of the charged capillary surface. We also observe an increase in the film thicknesses with increasing ionic strength in the PE solutions. This result is in good agreement with previously published works on LbL coating of planar substrates ${ }^{23,31,32}$.

In addition, we evaluate the morphology of PE layers by SEM micrographs of fibre cross-sections (Fig. 2b). For a moderate number of bilayers, salt-free PE solutions result in a relatively thin and smooth structure of the coating because of the self-adjustment of highly flexible polymeric chains $^{23}$ as well as the linear molecular conformation.

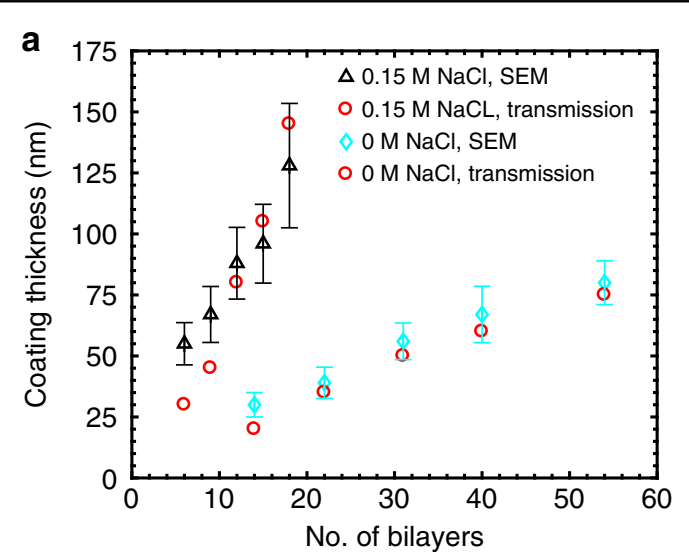

b

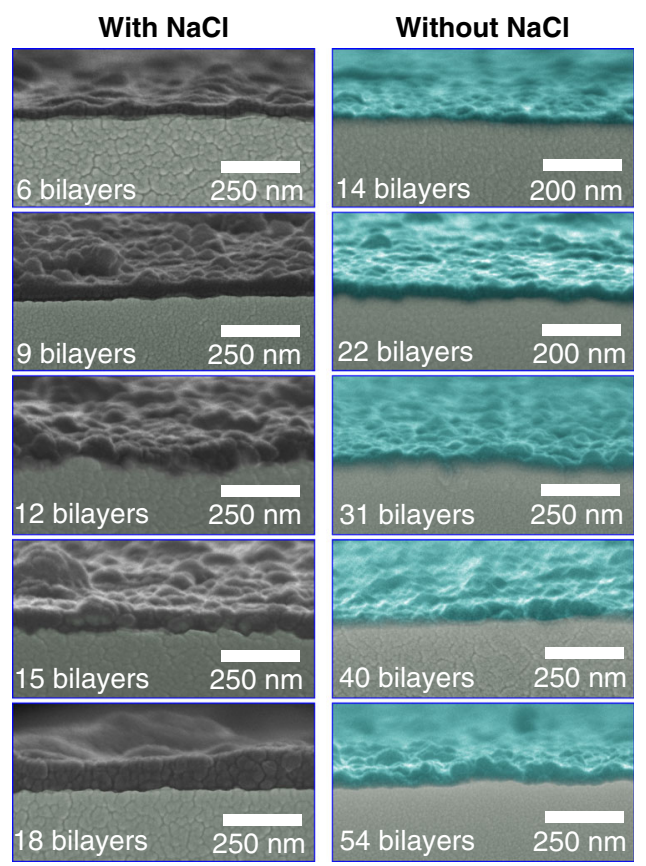

Fig. 2 Comparison of the capillary polymer coatings obtained by the PEs dissolved in deionized water and in buffer containing $0.15 \mathrm{M} \mathrm{NaCl}$. a Polymer coating thickness versus the number of PE bilayers. The coating thickness is evaluated from SEM images (SEM) and obtained by fitting the shifts in the fibre transmission spectra through the condition of Fabry-Perot resonances (transmission) (see the Supplementary information). Polymer coatings are included in the theoretical model as additional concentric layers of equal thickness on the core capillary. The thickness is fitted to obtain the coincidence of the minima positions for both the experimental and calculated transmission spectra. Error bars show the coating roughness. b SEM micrographs of the core capillaries functionalized with different numbers of PE bilayers. Left column: PEs in deionized water with $0.15 \mathrm{M} \mathrm{NaCl}$. Right column: PEs in pure deionized water. Pseudocolour indicates the polymer coating

With an increasing number of deposited PAH/PSS bilayers, quasi-spherical structures appear on the coating surface as a result of PE aggregation and lead to increased roughness, which, however, does not exceed $25 \mathrm{~nm}$ for the 
coating formed by 54 polymer bilayers. In contrast, the presence of $\mathrm{NaCl}$ in the PE solutions markedly modifies the structure of the polymeric molecules by a conformational transition from extended polymeric chains to globular structures, leading to the formation of polymer bundles with sizes up to hundreds of nanometres (Fig. 2b). Hence, PEs adsorbed from the saline buffer create a rigid quasi-spherical structure of coating with an average roughness of $50 \mathrm{~nm}$ for the coating formed by 18 PAH/PSS bilayers, giving rise to extra scattering for guiding light and enhancing the fibre optical losses. However, this coating does not significantly affect the transmission performance of functionalized HC-MOFs (Fig. S4 in the Supplementary information) since this roughness is much smaller than the light wavelength.

These results demonstrate that the structure of LbLassembled PAH/PSS films is highly dependent of the salt concentration in the PE solutions, and the coating thickness can be tailored in a reproducible and controllable way by varying the number of PE bilayers deposited. The salt-free PE solution provides the smallest roughness and finer tuning of the transmission windows, so we use $\mathrm{HC}$-MOFs functionalized with up to 54 bilayers by this protocol for IMOS.

It is instructive to note that LbL-assembled PAH/PSS films in HC-MOFs show extremely robust stability under various conditions, including long-term storage (Fig. S8), a wide temperature range from 22 to $120^{\circ} \mathrm{C}$ (Fig. S9), pH levels from 4 to 10 (Fig. S10) and various ionic strengths (Fig. S11) of the sample liquid along with subsequent washing of functionalized $\mathrm{HC}-\mathrm{MOF}$ by water and drying.

\section{Optical transmission of functionalized HC-MOFs}

HC-MOF functionalization leads to fine tuning of their optical transmission windows, as shown in Fig. 3. Specifically, shifts in maxima and minima of the transmission appear to be almost a linear function of the number of bilayers. This fact results from the similar RIs of the fibre glass $^{34,35}$ and $\mathrm{PAH} / \mathrm{PSS}^{36,37}$; thus, polymer coatings can be considered an effective instrument to vary the thickness of the core fibre capillary. Hence, the spectral positions of the transmission maxima and minima can be described by Eqs. (1) and (2), which, assuming a 1.8-nm thickness for every bilayer, yield the linear fits shown in Fig. 3c, d.

Importantly, LbL functionalization results in additional fibre losses. The results of cut-back measurements for the samples modified in the salt-free PE solution are presented in Fig. 4 (see also "Materials and methods"). One can observe that the polymer coating leads to an average extra attenuation of $\sim 0.02 \mathrm{~dB} \mathrm{~cm}^{-1}$ per single assembled PAH/PSS bilayer. However, this issue does not significantly disturb light guidance inside the fibre as long as the number of bilayers is limited.
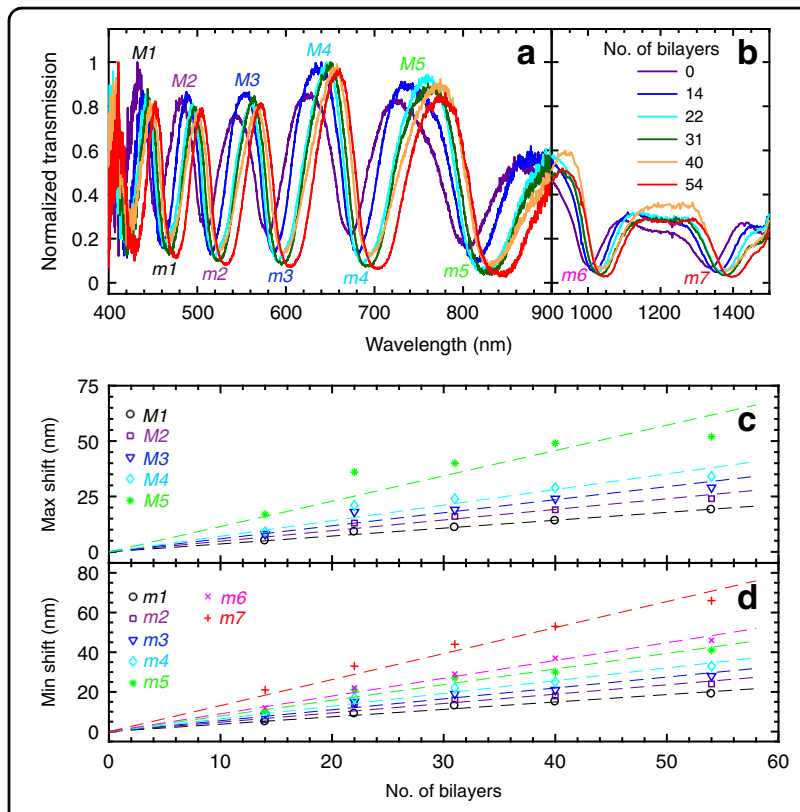

Fig. 3 Optical characterization of HC-MOFs functionalized by PEs adsorbed from the salt-free buffer. $\mathbf{a}, \mathbf{b}$ Transmission spectra in the visible $(400-900 \mathrm{~nm})$ and near-infra-red $(900-1500 \mathrm{~nm})$ ranges. The results for 6 samples with various numbers of PE bilayers are presented. Note the different scales along the $x$ axis for (a) and (b).

Shifts of maxima (c) and minima (d) of the transmission along with the linear fits

\section{IMOS in static mode}

We demonstrate IMOS by measurements of the RI for BSA dissolved in a water buffer with the help of six functionalized HC-MOFs with 0, 14, 22, 31, 40 and 54 PE bilayers. To simplify the interchange of fibres during the measurement, we replace the LCs in the setup (Fig. 1a) with a custom smart cuvette ${ }^{21}$ ("Material and methods"). We start with calibration of the system by recording the transmission of the water-filled fibre (Fig. 5a) and associate the spectral positions of the minima and the peak centroids with the water RIs by using Eqs. (1) and (2). Each fibre provides RIs at seven discrete wavelengths (four minima and three peak centroids) that are increased to 42 points due to the employment of six functionalized fibres with slightly shifted transmission windows. Thus, the validity of our approach is confirmed by the excellent agreement of our measurements with the well-known optical dispersion of deionized water adopted from ref. ${ }^{38}$ (Fig. 5b).

Figure 5a shows the evolution of transmission spectra caused by an increase in BSA concentration in a water buffer. Using these data, we extract RIs by the same technique as that for pure water filling and plot them along with the Sellmeier fits, as shown in Fig. 5b. To verify our results independently, we also measure the RIs of these samples by a multiwavelength Abbe refractometer 

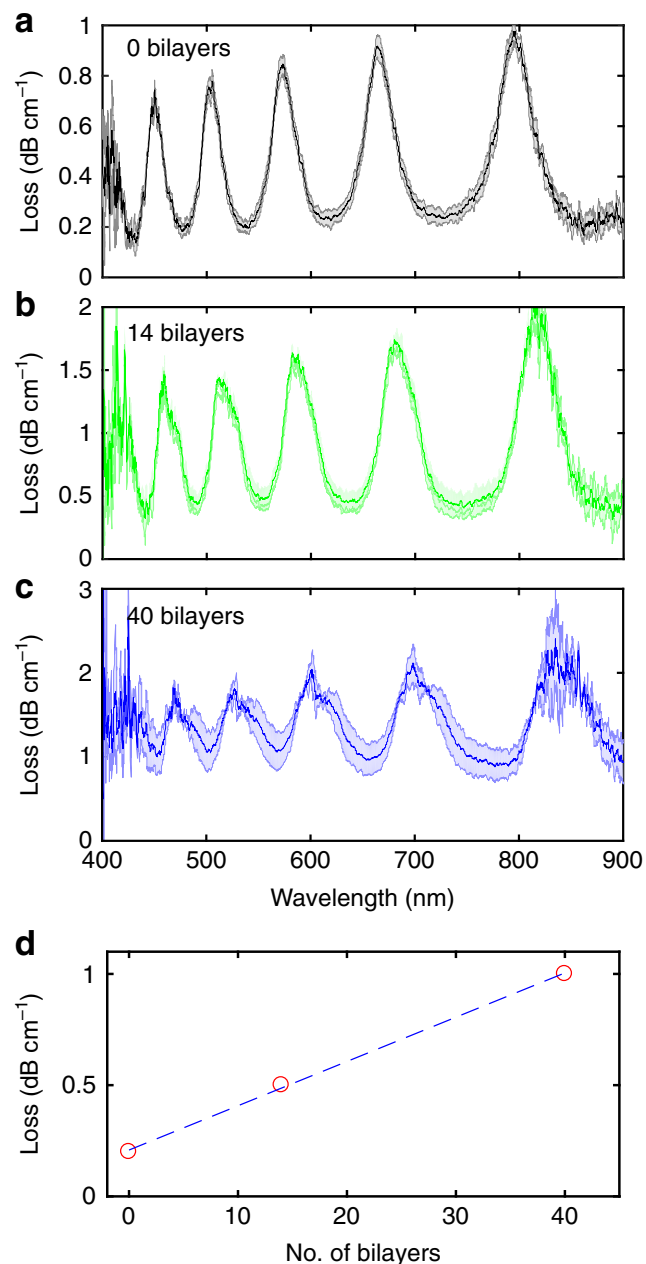

Fig. 4 Characterization of optical losses in functionalized HCMOFs by the cut-back technique. a Unmodified MOF. b, c Fibres coated with 14 and 40 PE bilayers in the salt-free solution. Shadow areas show the error range. Loss peaks fit Eq. (2). $\mathbf{d}$ The minimal optical loss in the transmission windows as a function of the bilayer quantity

(denoted by the stars in Fig. 5b) and find very good agreement with the IMOS results.

In practical applications, it is important not only to determine RIs of a biological liquid but also to quantify the concentration of target biomolecules. To this end, we calibrate our system by associating the RIs of the samples at different wavelengths with the concentration of BSA (Fig. 5c and Table S3). Since the concentration of BSA is quite low, these dependencies are linear.

However, it should be noted that solutions with strongly absorbing analytes can be investigated only with preliminary preparation of the probe via dilution. The higher optical losses introduced by the low-transparency filling media result in minima and maxima distortion (see the Supplementary information).

\section{RIS and FOM}

The key characteristics of label-free optical sensors are the RIS, defined as the ratio of the change in sensor output (the shift of the resonant wavelength) to the analyte RI variation, and the FOM, which normalizes the RIS to the width of the tracked resonance characterized by the full width at half maximum ${ }^{2}$. Using the fibre transmission spectra (Fig. 5a) and the determined RIs of the BSA-water solutions (Fig. 5b), we plot the transmission spectra minima versus the analyte RI (see Fig. $5 \mathrm{~d}$ for the longestwavelength minima and Fig. S15 for all minima). The slopes of the linear fits show the RIS of our sensor, which varies from $1100 \mathrm{~nm} \mathrm{RIU}^{-1}$ for blue light to $3000 \mathrm{~nm}$ $\mathrm{RIU}^{-1}$ for infra-red light. The corresponding FOM varies from 60 to $99 \mathrm{RIU}^{-1}$. These values along with the working spectral range are typical for surface plasmon and 2D material sensors ${ }^{2}$. However, our approach provides a great benefit of multispectral analysis in a broad spectral range.

\section{Optical dispersion of BSA}

It is important to note that the optical dispersion for a solution containing an investigated substance dissolved in a water buffer can be used to determine the optical dispersion of the pure product. Such a possibility is of special interest in cases when the substance of interest is difficult to synthesize in the form of thin films, which are normally suitable for analysis with standard techniques such as ellipsometry or refractometry. This is the case for BSA, whose RI has been reported for only a few wavelengths: $436^{39-42}, 546^{39,40,42}, 578^{42-44}, 589^{39,44}$ and $840 \mathrm{~nm}^{45}$. Accounting for BSA molecules as uniaxial ellipsoids with overall dimensions of $4 \mathrm{~nm} \times 4 \mathrm{~nm} \times 14 \mathrm{~nm}^{46,47}$, the extraction can be performed by using the effective medium approximation, which describes the effective permittivity $\varepsilon_{\text {eff }}$ of the solution by the Maxwell Garnett equation as follows ${ }^{48,49}$ :

$$
\varepsilon_{\mathrm{eff}}=\varepsilon_{\mathrm{e}}+\varepsilon_{\mathrm{e}} \frac{\frac{f_{\mathrm{BSA}}}{3} \sum_{j=x, y, z} \frac{\varepsilon_{\mathrm{i}}-\varepsilon_{\mathrm{e}}}{\varepsilon_{\mathrm{e}}+N_{j}\left(\varepsilon_{\mathrm{i}}-\varepsilon_{\mathrm{e}}\right)}}{1-\frac{f_{\mathrm{BSA}}}{3} \sum_{j=x, y, z} \frac{N_{j}\left(\varepsilon_{\mathrm{i}}-\varepsilon_{\mathrm{e}}\right)}{\varepsilon_{\mathrm{e}}+N_{j}\left(\varepsilon_{\mathrm{i}}-\varepsilon_{\mathrm{e}}\right)}}
$$

where $\varepsilon_{e}$ and $\varepsilon_{i}$ are the permittivities of the water buffer and the inclusions (BSA molecules), respectively, $f_{\mathrm{BSA}}$ is the volume fraction of the BSA molecules, and $N_{x}, N_{y}$ and $N_{z}$ are the depolarization factors over the $x, y$ and $z$ axes, respectively. The molecular weight of $66.5 \mathrm{kDa}^{46,47}$ and dimensions of BSA molecules allow us to translate the mass concentration of BSA into the volume filling factor $f_{\mathrm{BSA}}$. By using the known optical dispersion of water and the measured RIs of the BSA-water mixtures, we determine the optical dispersion of BSA (Fig. 6). To verify the repeatability of this process, we perform this procedure for three concentrations of BSA, $20 \mathrm{~g} \mathrm{~L}^{-1}$ $\left(f_{\mathrm{BSA}}=0.0213\right), 40 \mathrm{~g} \mathrm{~L}^{-1} \quad\left(f_{\mathrm{BSA}}=0.0425\right)$, and $60 \mathrm{~g} \mathrm{~L}^{-1}$ $\left(f_{\mathrm{BSA}}=0.0638\right)$, resulting in almost identical dispersion 

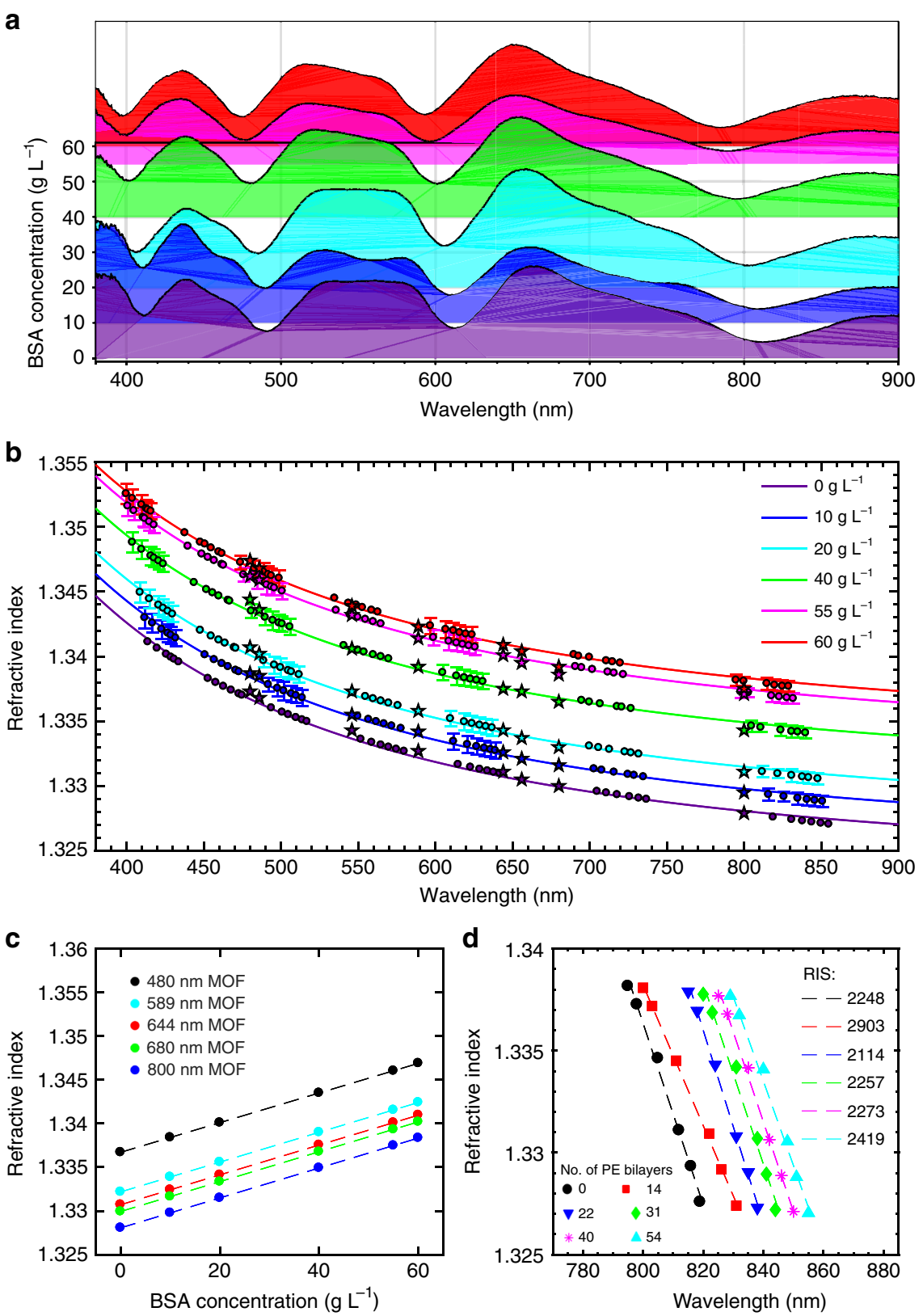

Fig. 5 Performance of IMOS for BSA dissolved in deionized water in static mode. a Transmission spectrum evolution with increasing BSA concentration for the non-modified HC-MOF. b Optical dispersion of BSA in a water buffer with different concentrations. The coloured points indicate the RI values extracted from the shifts in the minima and the peak centroids of the fibre transmission spectra, and the solid lines mark their Sellmeier fits. For pure water, the Sellmeier fit describes the water dispersion adopted from ref. ${ }^{38}$. The error bars are obtained from the spectrometer optical resolution. The stars mark Rls measured by the Abbe refractometer, provided for comparison. c Calibration relating the concentration of BSA and RIs of the BSA-water solution at different wavelengths. The RIs of BSA are extracted from the Sellmeier fits of the experimental points (see Table 1S), $\mathbf{d}$ The positions of the longest-wavelength minima as functions of RI extracted from (a) and (b). The error bars are smaller than the data points

curves. The discrepancy falls within the range of the measurement accuracy.

It is instructive to compare the RI dispersion of BSA obtained by IMOS with the data available in the literature $^{39-43,45}$ (Fig. 6). All previous measurements of BSA RI have been performed by refractometry of water-BSA solutions, and they show deviations from each other and from our results. However, the difference between the results is in the range of less than 0.025 RIU. These dissimilarities can be attributed to the distinct purity of BSA used in different measurements. In our experiments, we utilize BSA produced by Sigma-Aldrich with a purity 


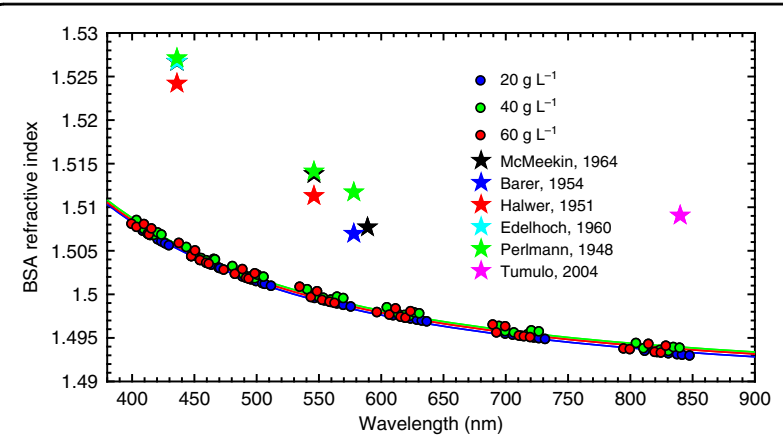

Fig. 6 The refractive index of BSA determined via the Maxwell Garnett approximation. Points correspond to the measurements of RI for BSA-water, and solid lines mark the Sellmeier fits (see the Supplementary Information, Table S4). The extraction was performed for three concentrations of BSA to show the repeatability of the result. The error bars are smaller than the marker size. The stars mark BSA RI values adopted from refs. ${ }^{39-43,45}$

above $96 \%$, while the purity of BSA measured in previous works was not reported. However, the purity is a critical parameter that can significantly affect the measurement results. To this end, we compare the results for BSA produced by Sigma-Aldrich ( $>96 \%$ purity $)^{50}$ (St. Louis, MO, USA) and Agat-Med (50\% purity) ${ }^{51}$ (Moscow, Russia) and find a discrepancy of $\sim 0.05$ RIU (Fig. S16). The purity of the samples has been verified by sodium dodecyl sulfate-polyacrylamide gel electrophoresis analysis (Figs. S17 and S18).

\section{IMOS in real time}

To demonstrate IMOS in a dynamic regime, we return to the setup where the HC-MOF is sealed in LCs (Fig. 1a). The experiment is organized as follows: we subsequently switch the peristaltic pump between seven solutions with different concentrations of BSA in a PBS buffer and use the specially developed algorithm in LabVIEW to track in real time the spectral positions of minima in the fibre transmission spectra. Figure 7 displays the results. Although the PBS buffer for BSA dissolution prevents the intensive formation of air bubbles while the analyte flows through the fibre, bubbles still appear at the moments of solution switching, leading to instantaneous disturbance of the minima positions during the transitional time intervals (coloured curves in Fig. 7). To circumvent this effect, we process the data by smoothing and present the results as black curves in Fig. 7. One can observe instantaneous shifts in the minima positions on the spot in response to variations in the analyte concentration. In turn, the analyte RIs can be determined via calibration in Fig. 5c. The repeatability of the measurements is evidenced by the two equal cycles of transitions.

In addition to exhibiting high repeatability, the dynamic test shows the real-time RI measurements in a wide spectral domain, and the response speed of the proposed sensor can be treated as instantaneously fast. However, considering the specific measurements, we should take into account the time needed for target molecules or particles to bind onto the inner surface of the fibre core. Thus, IMOS in real time is a powerful technique enabling instantaneous determination of the analyte optical dispersion and concentration. Fibre sealing into LCs connected to the peristaltic pump enables further integration into various optofluidic systems that demand fast, precise and easy sensing tools (Fig. 1). Considering their reusability and high stability to various environmental conditions, functionalized HC-MOFs can be used for the analysis of various biological liquids, and the cleaning of $\mathrm{HC}-\mathrm{MOF}$ can be ensured by the water flow, which removes the remaining solutions and adsorbed molecules.

It should be noted that real-time monitoring of RI optical dispersion simultaneously at 42 (or even several hundred) wavelengths can be achieved by using several post-processed HC-MOFs with necessary modifications of LCs and adaptation of the optical setup to parallel measurements (Figs. S5 and S6).

\section{Discussion}

In summary, we have proposed and experimentally demonstrated the technique of IMOS based on the analysis of spectral shifts in the minima and maxima of the HC-MOF transmission filled with a bioanalyte. In contrast to conventual optical biosensors that work at only a single wavelength, our approach allows simultaneous measurement of the RI at many wavelengths in the visible and near-infra-red spectral domains with RIS values up to $\sim 3000 \mathrm{~nm} \mathrm{RIU}^{-1}$ and FOMs reaching 99 $\mathrm{RIU}^{-1}$. Specifically, we have demonstrated the use of IMOS to measure the optical dispersion and concentration of BSA dissolved in water and PBS buffers in both static and dynamic regimes, with a resolution of $\sim 1 \mathrm{~g} \mathrm{~L}^{-1}$ when determining the BSA concentration, which matches the accuracy of the standard tests on albumin $^{18}$. Furthermore, for the first time, to our knowledge, we have extracted the RI of pure BSA at 42 spectral points in the wavelength range $400-850 \mathrm{~nm}$. Potentially, the number of wavelengths available for measurement of RI can be increased further to several hundred by producing the necessary number of $\mathrm{HC}$ MOFs with slightly shifted transmission windows. To this end, we have adapted the LbL technique to accurately coat fibre capillaries with polymer nanofilms. We have considered the effect of salt presence in the applied PE solutions on the coating performance and found that compared with the salt-enriched PEs, the salt-free PE solutions yield the smallest roughness and coating thickness per single bilayer. The proposed LbL deposition technique can be extended further, allowing one to reach novel sensing capabilities including $\mathrm{HC}-\mathrm{MOF}$ 
functionalization by specific molecules to capture biomolecules (DNA, antibodies, aptamers, Fab, etc.) and target particles ${ }^{52-54}$.

It is instructive to compare IMOS with refractometers and ellipsometers, which perform similar RI identification. The operational principle of conventional Abbe refractometers relies on using a set of filters, one filter per single acquisition wavelength, so that the RI measurements can be performed in a static regime only, and the number of wavelengths available for the measurements is restricted by the number of filters. Modern digital refractometers are more convenient, performing all operations in an automatic mode; however, they do not support real-time measurements. In-line refractometers, which are widely used in various manufacturing areas, target only a single wavelength (usually $589 \mathrm{~nm}$ ) and therefore cannot be used for the laboratory analysis of liquids over a wide spectral range. Overall, the modern market of available refractometers is diverse. However, these devices are expensive, restricted in the number of wavelengths available for measurement (typically less than 10) and cannot perform real-time measurements at several wavelengths in parallel.

The great advantage of ellipsometers is the ability to measure the RI in an ultrawide spectral band. However, ellipsometers are very expensive and bulky and require a complicated calibration process for every single measurement, so they cannot be easily integrated into any optofluidic or other sensing system to ensure real-time measurements.

Thus, the main benefits of IMOS in comparison to refractometers and ellipsometers are simplicity and cost efficiency, and the setup is quite compact and simple for reproduction. In addition, IMOS makes it possible to perform RI measurements over a wide spectral range, providing great capacity for practical use. Specifically, integration of LCs with surgery endoscopes will pave the way towards intraoperative analysis of bodily fluids, enabling surgeons to act in a timely manner under variable conditions. Multispectral analysis of saliva, urine, and ascitic fluid will facilitate diagnostics of various diseases.
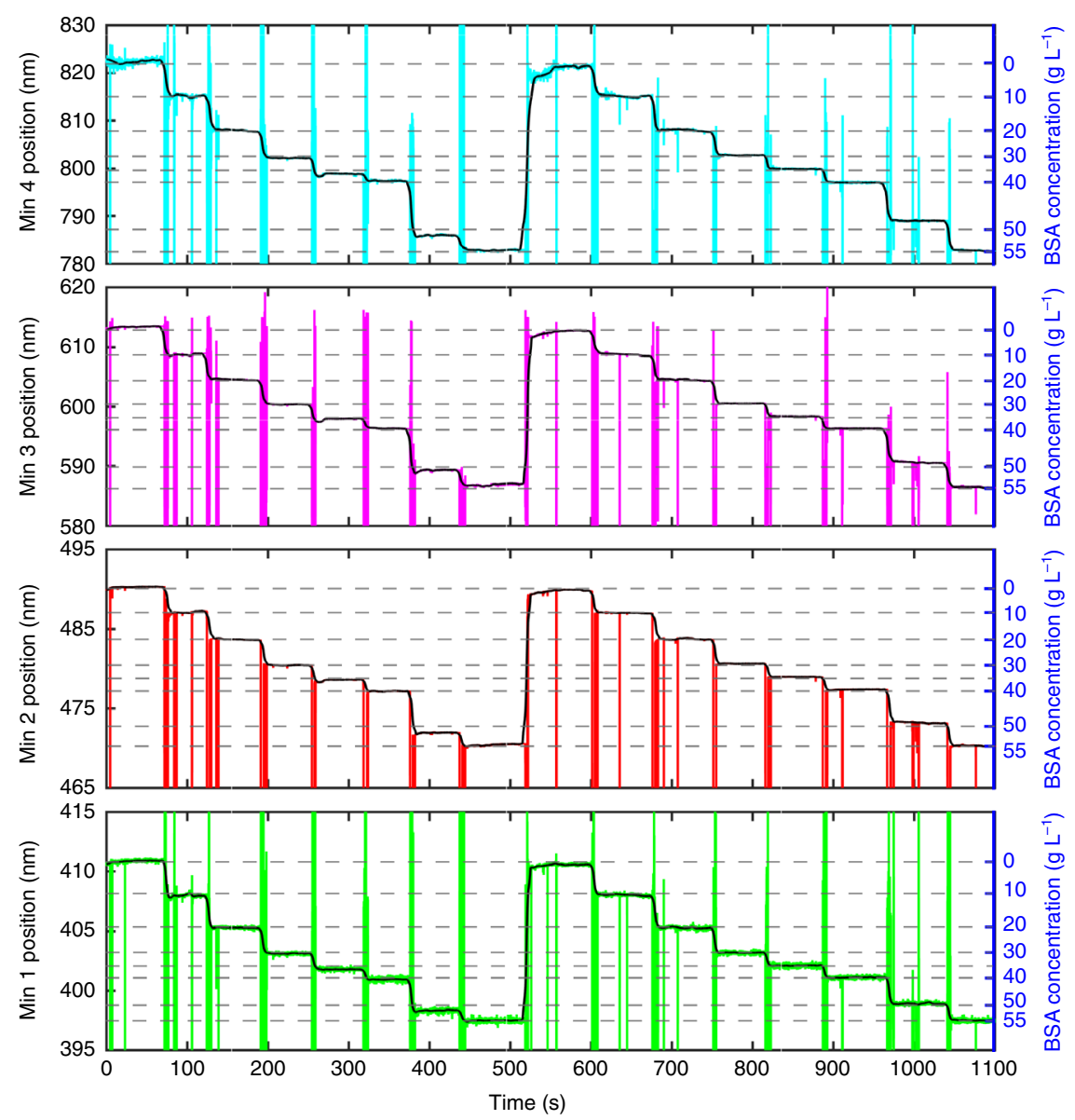

Fig. 7 IMOS in real time for BSA dissolved in PBS buffer. The coloured lines denote instantaneous positions of the transmission minima when the peristaltic pump is switched between five solutions with different concentrations of BSA. The black lines exhibit the data smoothed with MATLAB. The flow rate created by the peristaltic pump is fixed at $1 \mathrm{~mL} \mathrm{~min}{ }^{-1}$ 
In addition, IMOS enables accurate determination of the RI optical dispersion for various proteins and their complexes, which is important for reliable simulations of biological processes.

\section{Materials and methods HC-MOF sample fabrication}

We used HC-MOFs containing three concentric capillary layers surrounding a central hollow-core drawn from custom-made soft glass (Fig. 1b). The diameter of the central capillary is $\sim 240 \mu \mathrm{m}$, the outer diameter is $\sim 600 \mu \mathrm{m}$, the wall thickness of the first layer of capillaries is $1.82 \mu \mathrm{m}$, and the length of all samples is $6 \mathrm{~cm}$. The geometrical features of the $\mathrm{HC}-\mathrm{MOF}$ are detailed in Fig. S1. The spectral properties of these fibres have been discussed in refs. ${ }^{20,21}$.

\section{Chemical reagents}

All applied PEs and PBS were purchased from SigmaAldrich: PAH $(\mathrm{MW}=50,000)$, PSS $(\mathrm{MW}=70,000)$, and PEI $(M W=2,000,000)$. Deionized water was produced by a Millipore Milli-Q Plus 185 system. The BSA used in static dynamic measurements was supplied by Agat-Med and Sigma-Aldrich.

\section{Multilayered deposition process}

HC-MOFs were connected to a peristaltic pump (Shenchen) by a flexible silicone tube with an inner diameter of $1 \mathrm{~mm}^{22}$. To fix fibre samples, we produced special 3D-printed clamps, which ensured the efficient flow of solutions through the fibres. Our system supplies a highly controllable and persistent flow rate for any given solution capacity, allowing the uniform deposition of $\mathrm{PE}$ layers inside the capillaries. Prior to PE layer assembly, the fibres were washed with deionized water for $2 \mathrm{~min}$ with a speed of $500 \mu \mathrm{L} \mathrm{min}^{-1}$ to ensure that small dust particles were removed; then, the fibres were subjected to the LBL technique by a combination of inversely charged PEs (PAH/PSS) with a concentration of $2 \mathrm{mg} \mathrm{mL}^{-1}$ in both cases: in the presence of $0.15 \mathrm{M} \mathrm{NaCl}$ in the PE solution and in pure deionized water. The pump flow rate was set to $150 \mu \mathrm{L} \mathrm{min}^{-1}$, and the amount of PE solution used for a single PE layer formation was $1 \mathrm{~mL}$, which corresponded to an $\sim 7 \mathrm{~min}$ deposition cycle. Deionized water was applied after each deposited PE layer with a speed of $200 \mu \mathrm{L} \mathrm{min}{ }^{-1}$ for $2 \mathrm{~min}$ to wash the samples and to remove unadsorbed molecules. The very first bilayer of PEI/PSS was followed by the desired number of PAH/PSS bilayers to finalize the coating formation.

\section{Optical characterization of functionalized HC-MOFs}

The output light of a broadband halogen lamp (Thorlabs SLS201L, 360-2600 nm) was initially collimated
(Thorlabs F220SMA-532) and then focused by a $10 \times$ objective (Olympus) to the fibre input. The other $10 x$ objective (Olympus) was used to collect the transmitted light, which was further guided to either a compact CCD spectrometer (Ocean Optics QE Pro) operating in the extended wavelength region $(350-1000 \mathrm{~nm})$ or an IR spectrometer (Ocean View NIRQuest). All of the transmission spectra were initially normalized to the spectrum of the halogen lamp and then to its maximum value. A colour CCD camera (Thorlabs DCU223C) in the collection part was installed to control the coupling conditions and the fibre end face cleaving quality and to record the output mode profile. The analysis of the losses induced by deposited PE layers was performed by the cut-back method. The light was coupled into the fibre, and four cut-back steps were performed with a 6-cm-long sample under fixed coupling. The purity of the fundamental mode was controlled by the CCD camera.

\section{Multispectral sensing of albumin solutions}

In the static regime, $\mathrm{HC}$-MOFs were integrated into a smart cuvette and filled by the solutions in the test through capillary action. Once the fibre was filled completely and no air bubbles appeared, the transmission spectrum was recorded successively for each fibre sample. In the dynamic regime, the fibre was sealed in specially designed LCs. A detailed schematic of the designed LCs is illustrated in Fig. S14. Liquid samples were streamed through the HC-MOFs by the peristaltic pump connected to the inlet of the LC. Real-time tracking of the transmission spectra minima was achieved by means of LabVIEW software. We used two spectrometers (Ocean Optics QE Pro) to cover the visible and IR spectral ranges.

\section{BSA refractometric measurements}

The control measurement of RI for BSA (Fig. 6) was carried out by a multiwavelength Abbe refractometer (DR-M2/1550, Atago, Japan). The multiwavelength Abbe refractometer allows one to measure the RI in the wavelength range of $450-1550 \mathrm{~nm}$ with an accuracy of \pm 0.0002 . As the source of radiation, we used a high-power incandescent lamp. The wavelength of light was determined by narrow band-pass filters with transmission windows of $480 \pm 2 \mathrm{~nm}, 486 \pm 2 \mathrm{~nm}, 546 \pm 2 \mathrm{~nm}, 589 \pm$ $2 \mathrm{~nm}, 644 \pm 2 \mathrm{~nm}, 656 \pm 2 \mathrm{~nm}, 680 \pm 5 \mathrm{~nm}$ and $800 \pm 5 \mathrm{~nm}$. At the beginning of the experiment, the device was calibrated by measuring the RI of the prism $(n=1.3327)$ at a wavelength of $589 \mathrm{~nm}$ (the absorption band of sodium). Monobromonaphthalene was used as a contact liquid. The average measurement error of the RI was \pm 0.0003 . The prism temperature during the measurements was kept at $+24{ }^{\circ} \mathrm{C}$ by means of water circulation in the refractometer. 


\section{Acknowledgements}

This work was supported by the Russian Foundation for Basic Research (RFBR grant no. 19-32-90249). E.N.L. and V.V.T. were supported by RFBR grant no. 1852-16025. S.V.G., O.V.S., T.S.Z. and D.A.G. were supported by RFBR grant no. 1829-08046. This work was supported in part by the Ministry of Science \& Technology of Israel (grant no. 79518).

\section{Author details}

'Skolkovo Institute of Science and Technology, 3 Nobelya str., Moscow 121205, Russia. ${ }^{2}$ Department of Electrical Engineering, Tel Aviv University, Ramat Aviv, Tel Aviv 69978, Israel. 'Light-Matter Interaction Centre, Tel Aviv University, Ramat Aviv, Tel Aviv 69978, Israel. ${ }^{4}$ Saratov State University, 83 Astrakhanskaya str., Saratov 410012, Russia. ${ }^{5}$ Tomsk State University, 36 Lenin's av., Tomsk 634050, Russia. ${ }^{6}$ M.V. Lomonosov Moscow State University, Leninskie Gory, 1-3, Moscow 119992, Russia. ${ }^{7}$ SPE LLC Nanostructured Glass Technology, 10150 Let Oktjabrja, Saratov 410033, Russia. ${ }^{8}$ Center for Photonics and 2D Materials, Moscow Institute of Physics and Technology, Dolgoprudny 141700, Russia. ${ }^{9}$ Institute of Precision Mechanics and Control of the Russian Academy of Sciences, 24 Rabochaya str., Saratov 410028, Russia

\section{Author contributions}

D.A.G., R.E.N. and P.G.L. conceived and designed the research. T.E. performed the experiments. R.E.N. designed the computational model. R.E.N. and T.E. performed the theoretical analysis. A.A.M. contributed to the optical measurements. I.G. contributed to the extraction of refractive indexes of BSA inclusions, automatization of the optical setup and data curation in the realtime measurements. O.V.S. analyzed the BSA samples. D.A.G., P.G.L., R.E.N. and T.E. analyzed the data. S.V.G. fabricated the liquid cells and special fibre holders. V.A. and S.S.K. performed SEM measurements. E.N.L. performed refractometric measurements of BSA solutions with the Abbe refractometer. J.S.S. fabricated HC-MOFs. R.E.N., T.E., and D.A.G. wrote the manuscript. T.S.Z., V.V.T., P.G. and P.G.L. contributed to the manuscript preparation. T.E. and S.V.G. prepared the graphics. D.A.G. and R.E.N. supervised the project. All authors contributed to the research activities.

\section{Conflict of interest}

The authors declare that they have no conflict of interest.

Supplementary information is available for this paper at https://doi.org/ 10.1038/s41377-020-00410-8.

Received: 12 May 2020 Revised: 17 September 2020 Accepted: 24 September 2020

Published online: 10 October 2020

\section{References}

1. Quesada-González, D. \& Merkoçi, A. Nanomaterial-based devices for point-ofcare diagnostic applications. Chem. Soc. Rev. 47, 4697-4709 (2018).

2. $\mathrm{Xu}, \mathrm{Y}$. et al. Optical refractive index sensors with plasmonic and photonic structures: promising and inconvenient truth. Adv. Optical Mater. 7, 1801433 (2019).

3. Chen, G. Y. et al. Nanochemistry and nanomedicine for nanoparticle-based diagnostics and therapy. Chem. Rev. 116, 2826-2885 (2016).

4. Cubillas, A. M. et al. Photonic crystal fibres for chemical sensing and photochemistry. Chem. Soc. Rev. 42, 8629-8648 (2013).

5. Farka, Z. et al. Nanoparticle-based immunochemical biosensors and assays: recent advances and challenges. Chem. Rev. 117, 9973-10042 (2017).

6. Shandilya, R. et al. Nanobiosensors: point-of-care approaches for cancer diagnostics. Biosens. Bioelectron. 130, 147-165 (2019).

7. Sreekanth, K. V. et al. Extreme sensitivity biosensing platform based on hyperbolic metamaterials. Nat. Mater. 15, 621-627 (2016).

8. Yesilkoy, F. et al. Ultrasensitive hyperspectral imaging and biodetection enabled by dielectric metasurfaces. Nat. Photonics 13, 390-396 (2019).

9. Wang, Z. Tissue refractive index as marker of disease. J. Biomed. Opt. 16, 116017 (2011)

10. Mazarevica, G., Freivalds, T. \& Jurka, A. Properties of erythrocyte light refraction in diabetic patients. J. Biomed. Opt. 7, 244 (2002)
11. Goldwasser, P. \& Feldman, J. Association of serum albumin and mortality risk. J. Clin. Epidemiol. 50, 693-703 (1997).

12. Liu, Z. B. \& Chen, X. Y. Simple bioconjugate chemistry serves great clinical advances: albumin as a versatile platform for diagnosis and precision therapy. Chem. Soc. Rev. 45, 1432-1456 (2016).

13. Lazareva, E. N. \& Tuchin, V. V. Measurement of refractive index of hemoglobin in the visible/NIR spectral range. J. Biomed. Opt. 23, 1-9 (2018).

14. Zysk, A. M., Chaney, E. J. \& Boppart, S. A. Refractive index of carcinogeninduced rat mammary tumours. Phys. Med. Biol. 51, 2165-2177 (2006).

15. Zhernovaya, O. S., Tuchin, V. V. \& Meglinski, I. V. Monitoring of blood proteins glycation by refractive index and spectral measurements. Laser Phys. Lett. 5, 460-464 (2008).

16. Lee, S. et al. Refractive index tomograms and dynamic membrane fluctuations of red blood cells from patients with diabetes mellitus. Sci. Rep. 7, 1039 (2017).

17. Busher, J. T. Serum albumin and globulin. In Clinical Methods: The History Physical, and Laboratory Examinations (eds. Walker, H. K. et al.) 479-499 (Butterworths, 1990).

18. Duly, E. B. et al. Measurement of serum albumin by capillary zone electrophoresis, bromocresol green, bromocresol purple, and immunoassay methods. J. Clin. Pathol. 56, 780-781 (2003).

19. Yu, W. Y. et al. Cavity optomechanical spring sensing of single molecules. Nat Commun. 7, 12311 (2016).

20. Zheltikov, A. M. METHODOLOGICAL NOTES: Colors of thin films, antiresonant phenomena in optical systems, and the limiting loss of modes in hollow optical waveguides. Phys. Uspekhi 51, 591-600 (2008).

21. Noskov, R. E. et al. Enabling magnetic resonance imaging of hollow-core microstructured optical fibers via nanocomposite coating. Opt. Express 27, 9868-9878 (2019).

22. Ermatov, T. et al. Microstructured optical waveguide-based endoscopic probe coated with silica submicron particles. Materials 12, 1424 (2019).

23. Zhang, L., Guo, H. Y. \& Sun, J. Q. Salt effects on the structural tailoring of layerby-layer assembled polyelectrolyte complexes and salt-containing polyelectrolyte films. Thin Solid Films 653, 258-266 (2018).

24. Kolasińska, M. \& Warszyński, P. The effect of support material and conditioning on wettability of PAH/PSS multilayer films. Bioelectrochemistry $\mathbf{6 6 , 6 5 - 7 0 ~ ( 2 0 0 5 ) . ~}$

25. Kolasińska, M. \& Warszyński, P. The effect of nature of polyions and treatment after deposition on wetting characteristics of polyelectrolyte multilayers. Appl. Surf. Sci. 252, 759-765 (2005).

26. Decher, G. Fuzzy nanoassemblies: toward layered polymeric multicomposites. Science 277, 1232-1237 (1997)

27. De Villiers, M. M. et al. Introduction to nanocoatings produced by layer-bylayer (LbL) self-assembly. Adv. Drug Deliv. Rev. 63, 701-715 (2011).

28. Hoogeveen, N. G. et al. Formation and stability of multilayers of polyelectrolytes. Langmuir 12, 3675-3681 (1996).

29. Neff, P. A. et al. Electrical detection of self-assembled polyelectrolyte multilayers by a thin film resistor. Macromolecules 39, 463-466 (2006).

30. Arys, X. et al. Ultrathin multilayers made by alternate deposition of ionenes and polyvinylsulfate: From unstable to stable growth. Thin Solid Films 327-329, 734-738 (1998).

31. El Haitami, A. E. et al. Effect of the supporting electrolyte anion on the thickness of PSS/PAH multilayer films and on their permeability to an electroactive probe. Langmuir 25, 2282-2289 (2009).

32. Feldötö, Z., Varga, I. \& Blomberg, E. Influence of salt and rinsing protocol on the structure of PAH/PSS polyelectrolyte multilayers. Langmuir 26, 17048-17057 (2010)

33. Toda, M. et al. Surface stress, thickness, and mass of the first few layers of polyelectrolyte. Langmuir 24, 3191-3198 (2008).

34. Kang, S., Day, D. E. \& Stoffer, J. O. Measurement of the refractive index of glass fibers by the Christiansen-Shelyubskii method. J. Non-Crystalline Solids 220, 299-308 (1997).

35. Tompkins, H. G., Smith, S. \& Convey, D. Optimizing the ellipsometric analysis of a transparent layer on glass. Surf. Interface Anal. 29, 845-850 (2000).

36. Wang, D. et al. Layer-by-layer assembled transparent polymeric adhesive films with adjustable refractive indices. Int. J. Adhes. Adhesives 85, 202-207 (2018).

37. Kim, J. H., Hwang, J. H. \& Lim, T. Y. A layer-by-layer self-assembly method for organic-inorganic hybrid multilayer thin films. J. Ceram. Process. Res. 10, 770-773 (2009).

38. Daimon, M. \& Masumura, A. Measurement of the refractive index of distilled water from the near-infrared region to the ultraviolet region. Appl. Opt. 46, 3811-3820 (2007) 
39. McMeekin, T. L., Groves, M. L. \& Hipp, N. J. Refractive indices of amino acids, proteins, and related substances. In Amino Acids and Serum Proteins (ed. Stekol, J. A.) 54-66 (Washington: American Chemical Society, 1964).

40. Halwer, M., Nutting, G. C. \& Brice, B. A. Molecular weight of lactoglobulin, ovalbumin, lysozyme and serum albumin by light scattering. J. Am. Chem. Soc. 73, 2786-2790 (1951).

41. Edelhoch, H. The properties of thyroglobulin. I. The effects of alkali. J. Biol. Chem. 235, 1326-1334 (1960).

42. Perlmann, G. E. \& Longsworth, L. G. The specific refractive increment of some purified proteins. J. Am. Chem. Soc. 70, 2719-2724 (1948)

43. Barer, R. \& Tkaczyk, S. Refractive index of concentrated protein solutions. Nature 173, 821-822 (1954).

44. Huglin, M. B. Specific refractive index increments of polymer solutions. Part I. Literature values. J. Appl. Polym. Sci. 9, 3963-4001 (1965).

45. Tumolo, T., Angnes, L. \& Baptista, M. S. Determination of the refractive index increment $(d n / d c)$ of molecule and macromolecule solutions by surface plasmon resonance. Anal. Biochem. 333, 273-279 (2004).

46. Wright, A. K. \& Thompson, M. R. Hydrodynamic structure of bovine serum albumin determined by transient electric birefringence. Biophys. J. 15, 137-141 (1975).
47. Peters, Jr. T. J. The Albumin molecule: its structure and chemical properties. In All About Albumin. Biochemistry, Genetics, and Medical Applications, 9-75 (Academic Press, San Diego, 1996).

48. Levy, O. \& Stroud, D. Maxwell Garnett theory for mixtures of anisotropic inclusions: application to conducting polymers. Phys. Rev. B 56, 8035-8046 (1997).

49. Sihvola, A. Electromagnetic Mixing Formulas and Applications (Institute of Electrical Engineers, London, 1999).

50. Sigma-Aldrich. A2153-Bovine Serum Albumin (Sigma-Aldrich, 2020). https:// www.sigmaaldrich.com/catalog/product/sigma/a2153?lang=en\&region=RU.

51. Agat-Med. Bilirubin Etalon Agat. (Agat-Med, 2020). https://www.agat.ru/ documents/instructions/5075/.

52. Barozzi, M. et al. Optical fiber sensors for label-free DNA detection. J. Lightwave Technol. 35, 3461-3472 (2017).

53. Dinish, U. S. et al. Sensitive multiplex detection of serological liver cancer biomarkers using SERS-active photonic crystal fiber probe. J. Biophotonics 7, 956-965 (2014).

54. Dinish, U. S. et al. Highly sensitive SERS detection of cancer proteins in low sample volume using hollow core photonic crystal fiber. Biosens. Bioelectron. 33, 293-298 (2012) 